\title{
Witness the fitness
}

For reasons of history and convenience, gross domestic product (GDP) has become the default measure of useful economic activity, and generating more of it is the target of every nation. This despite economists' wide acknowledgement (J. E. Stiglitz, A. Sen and J.-P. Fitoussi, Report by the Commission on the Measurement of Economic Performance and Social Progress; 2009) that GDP is a terrible indicator of beneficial activity. It says nothing about social well-being, or inequality, and goes up if more pollution requires more paid work to clean it up. An alternative measure known as the genuine progress indicator (GPI) includes a number of social and environmental factors, and gives a rather different picture of the benefits of growth. For the US, GPI data indicate that economic growth made the nation better off up to around 1970, but gains since then have been fully offset by worsening social conditions and the depletion of natural resources.

Even so, predicting GDP remains important, as this partial measure does have a direct bearing on key human variables internal to any economy - things like employment, inflation and interest rates. Numerable independent firms as well as official bodies including the International Monetary Fund (IMF) routinely predict GDP to help governments and businesses plan for the future. They've achieved limited success linking GDP to a range of socially and economically relevant aggregate variables such as labour productivity, education, investment or national debt. In the past few years, however, physicists have brought an important new perspective to the problem, and achieved an impressive improvement in prediction accuracy by going beyond aggregate measures.

The technique, inspired by work in complexity science, looks in detail at the way nations are linked together by the complex network of products they export. Writing in Nature Physics, Andrea Tacchella et al. take this complex network perspective several steps further, reporting a forecasting scheme that outperforms five-year IMF forecasts by more than 25\% (Nat. Phys. https://doi.org/10.1038/ s41567-018-0204-y; 2018). Moreover, they present evidence that the data on which their analyses rest capture core aspects of GDP progression that are truly complementary to those in traditional methods.

Building on earlier research, the authors pursue a self-consistent calculation something akin to Google's PageRank algorithm - to determine both the economic 'fitness' of each nation, as well as the 'complexity' of each exported product. In this case, each nation's 'fitness' is defined as a weighted sum over that country's exports, with each product's 'complexity' giving the weight. That is, the more different things a country produces, and the more complex those things are, the greater its fitness. In turn, a product's complexity is determined mathematically by how few countries can successfully make it. The most complex products are made by only the few most advanced nations. The authors define a set of equations from which the nations' fitnesses and product complexities can be self-consistently calculated.

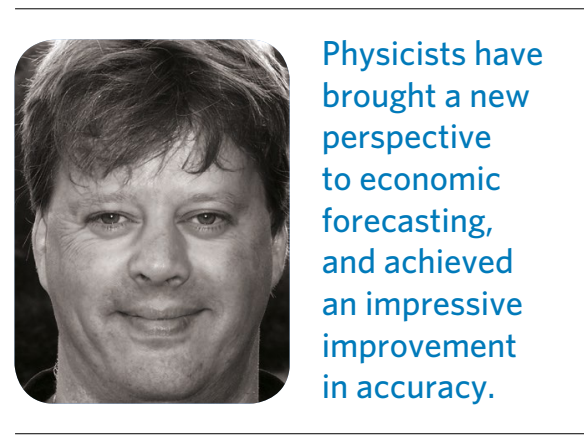

As it turns out, the results indicate that fitness tends to be roughly correlated to GDP per capita. However, it's the deviations from this relation that carry hidden information useful for forecasting. If a nation is poorer in GDP terms than its fitness score suggests, one might expect it to soon get richer - after all, it has the technical capabilities. The data indicates a flow in the two-dimensional phase plane fitness-GDP, and predictions come from projecting this flow forward. Although some of the flow is quite noisy, a region of predictable flow includes many nations, particularly relatively advanced nations that have become capable of producing many sophisticated products: the capacity shows up in the fitness measure even before it affects GDP. China, Vietnam and India, for example, all look poised for further growth.

These results were achieved in earlier work. The new paper takes several further steps forward. For example, as the calculation rests entirely on international data on the network of exported product, Tacchella et al. have taken pains to clean and regularize the raw data from UN COMTRADE, a trade statistics repository maintained by the UN. As they show, the use of this improved data significantly boosts the forecasting power of technique. In particular, compared to earlier studies (including the authors' own work), this procedure vastly extends the set of 'predictable' countries, as previously noisy regions of the flow become more regular.

The paper makes other advances also. For one thing, it shows that predictions improve by including some momentum - recent GDP growth implies further similar growth. Predictions are also more valuable if they come with some reliable estimation of their expected uncertainty, and the improved method precisely reports the uncertainty of each prediction. The authors tested these estimates, finding excellent consistency with the true out-of-sample error.

On another, more technical level, this paper for the first time carries out the model construction in a fully out-of-sample way, always using only past data to make predictions looking forward. Specifically, the authors made 482 GDP growth forecasts on three distinct five-year windows: 20082013, 2009-2014 and 2010-2015. In each case, they performed the data sanitation using only data up to the beginning of the forecasting window. This makes for a fair and unbiased comparison with historical IMF forecasts.

Finally, Tacchella et al. were also able to show definitively that this new approach isn't just a minor twist on traditional methods, but a fundamentally distinct approach that captures entirely new information of predictive value. In a comparison of forecasting errors by the new and traditional methods, they found that errors made by the dynamical systems approach were strongly uncorrelated with those made by IMF models. This suggests that the two models capture essentially different kinds of useful information, which implies that future improvements may be found merely by finding ways to combine the two approaches.

Of course, this is only a beginning, and there remains great scope for further improvement. Perhaps this method, or generalizations of it, may be used to look in more detail at measure such as genuine progress, and find ways to measure fitness on that score as well.

\section{Mark Buchanan}

Published online: 30 July 2018 https://doi.org/10.1038/s41567-018-0245-2 